\title{
Electrocardiographic measures of ventricular repolarisation dispersion in patients with coronary artery disease susceptible to ventricular fibrillation
}

\author{
L Oikarinen, L Toivonen, M Viitasalo
}

\begin{abstract}
Objective-To study electrocardiographic measures of ventricular repolarisation dispersion in patients prone to ventricular fibrillation compared with controls matched for the extent of coronary heart disease and the use of $\beta$ blockers.

Design-A case-control study.

Setting-Cardiovascular laboratory of a tertiary referral centre.

Patients-Fifty patients with documented ventricular fibrillation not associated with acute myocardial infarction, and their controls matched for sex, age, number of diseased coronary vessels, left ventricular ejection fraction, previous myocardial infarction and its location, and the use of $\beta$ blockers.
\end{abstract}

Main outcome measures-Electrocardiographic measures of $Q T, J T$, and $T_{\text {end }}$ interval dispersions in a 12 lead electrocardiogram.

Results-The ventricular fibrillation patients compared to controls showed increased mean (SD) $\mathbf{Q T}_{\text {apex }}$ dispersion (53 (18) $\mathrm{ms} v 44$ (18) ms, respectively; $\mathrm{p}<0.01)$ and mean (SD) $T_{\text {end }}$ dispersion (46 (17) ms $v 38$ (15) ms, respectively; p < 0.05).

Conclusions-Increased $Q T_{\text {apex }}$ and $T_{\text {end }}$ dispersions are associated with a susceptibility to ventricular fibrillation even when the extent of the coronary heart disease and use of $\beta$ blockers are taken into consideration. However, because of a considerable overlap between the groups, measures of QT dispersion assessed from a 12 lead electrocardiogram do not provide clinically useful information for identification of patients at risk of sudden cardiac death.

(Heart 1998;79:554-559)

Keywords: QT dispersion; ventricular fibrillation; coronary artery disease

Department of Medicine, Division of

Cardiology, Helsinki

University Central

Hospital,

Haartmaninkatu 4, 00290 Helsinki, Finland

L Oikarinen

L Toivonen

M Viitasalo

Correspondence to:

Dr Oikarinen.

Accepted for publication 24 February 1998

The high incidence of sudden cardiac death in patients with coronary artery disease and development of new treatment strategies for these patients challenges the clinician to detect patients at risk. In most of these patients the mechanism of sudden cardiac death is ventricular fibrillation. ${ }^{1}$ Experimental studies have provided strong evidence for the pathophysiological mechanism of dispersion of ventricular recovery time for the genesis of ventricular fibrillation. $^{2-4}$ In experimental studies, the excitability is proportional to the duration of repolarisation and, thus, dispersion of refractoriness parallels dispersion of repolarisation. ${ }^{2}$ Invasive studies in the human right ventricle have confirmed the strong correlation between action potential duration and the effective refractory period with different cycle lengths at a given endocardial site. ${ }^{5}$ However, for clinical studies, these invasive measurements are impractical.

Day and colleagues ${ }^{6}$ first proposed that interlead variability of QT intervals in 12 lead electrocardiogram (ECG), QT dispersion, reflects dispersion of ventricular recovery time, thus providing a convenient tool for clinical studies. This hypothesis has received strong support from animal experiments ${ }^{7}$ and invasive studies in man. ${ }^{8}$ In clinical studies, increased QT dispersion has been associated with susceptibility to malignant ventricular arrhythmias in long QT syndrome, ${ }^{6}$ hypertrophic cardiomyopathy, ${ }^{9}$ mitral valve prolapse,${ }^{10}$ patients operated on for tetralogy of Fallot, ${ }^{11} 12$ and coronary artery disease. ${ }^{13-15}$ In coronary artery disease, increased QT dispersion has been associated with ventricular fibrillation in acute myocardial infarction. ${ }^{13}$ However, the role of QT dispersion has not been established in detecting patients who present with ventricular fibrillation not associated with acute myocardial infarction. Furthermore, electrocardiographic dispersion measures other than the conventional QT dispersion (measured to the end of the $T$ wave) might better reveal dispersion of ventricular recovery time. ${ }^{7}$

Our study was designed to test whether electrocardiographic measures of dispersion of ventricular repolarisation can identify patients with coronary artery disease who are at risk of ventricular fibrillation when the extent of the coronary heart disease is taken into consideration.

\section{Methods}

STUDY PATIENTS

Patients with coronary artery disease referred to Helsinki University Central Hospital during 1988-95 for an electrophysiological study because of successful resuscitation from ventricular fibrillation were considered as cases for our study. The following inclusion criteria were used: ventricular fibrillation documented on an electrocardiographic chart strip; the ventricular fibrillation was not associated with acute myocardial infarction (no appearance of new $Q$ waves in the electrocardiogram or a significant rise in the $\mathrm{MB}$ fraction of creatinine kinase 
enzyme); and patients had coronary artery disease documented by coronary angiography without evidence of any other significant heart disease. Patients meeting these criteria were excluded if the electrophysiologist suspected proarrhythmia as the cause of ventricular fibrillation. If the registered ECG had less than eight measurable $\mathrm{QT}_{\text {end }}$ intervals, showed any rhythm other than sinus rhythm, or showed a complete bundle branch block, the patient was also excluded. A total of 50 patients met the criteria and were included in our study (ventricular fibrillation group). None of these patients had experienced a known previous episode of sustained monomorphic ventricular tachycardia. Three of these patients were on mexiletine treatment at the time of the ventricular fibrillation because of frequent ventricular extrasystoles.

The control group comprised patients with coronary artery disease referred for coronary angiography during the same time period. For each patient in the ventricular fibrillation group, a control patient was matched for sex, age ( \pm 10 years), number of diseased coronary vessels in coronary angiography, left ventricular ejection fraction ( $\pm 10 \%$ units), history, and location of a previous myocardial infarction as well as $\beta$ blockers during the registered ECG. Controls were required to have coronary artery disease (documented in the angiography) without evidence of any other significant heart disease and no history of syncope or ventricular tachyarrhythmias. No class I or III antiarrhythmic drugs were allowed. The criteria for the registered ECG were the same as for the ventricular fibrillation group.

STUDIES OF THE CORONARY ARTERY DISEASE In routine coronary angiograms, a luminal stenosis $\geqslant 50 \%$ was considered significant. The left ventricular ejection fraction was determined from contrast left ventriculography in the right anterior oblique projection using the area-length method. Q waves in the ECG and the presence of akinetic or dyskinetic myocardial wall segments in the ventriculography were used to evaluate the location of the previous myocardial infarction. A left ventricular aneurysm was defined as a dyskinetic segment of myocardium producing a distinct distortion of the diastolic left ventricular contour. Bicycle exercise tests were carried out starting from a load of $50 \mathrm{~W}$ and increasing it by $25-50 \mathrm{~W}$ every three minutes. The test was considered positive if there was a depression of the ST segment $\geqslant 0.1 \mathrm{mV}$ measured at $80 \mathrm{~ms}$ after the $\mathrm{J}$ point or an anginal chest pain.

ELECTROPHYSIOLOGICAL STUDY

All patients in the ventricular fibrillation group underwent programmed electrophysiological stimulation. The median time interval from the ventricular fibrillation to the programmed electrophysiological stimulation was 21 days (the shortest time interval was six days). The protocol included stimulation from two right ventricular sites with two drive cycle lengths and use of up to three extra stimuli. An induced monomorphic ventricular tachycardia was de- fined as sustained if it lasted for longer than 30 seconds or required overdrive pacing or cardioversion before that time because of haemodynamic collapse.

\section{ELECTROCARDIOGRAPHIC MEASUREMENTS}

ECG intervals

The ECGs were registered at rest before the programmed electrophysiological stimulation in the ventricular fibrillation group and before coronary angiography in the control group during the same hospital stay. If class I or III antiarrhythmic drugs had been started after ventricular fibrillation, they had to be discontinued at least five drug half lives before the registered ECG or electrophysiological study. The paper speed was $50 \mathrm{~mm} / \mathrm{second}$ and the calibration was $10 \mathrm{~mm} / \mathrm{mV}$ in the registered ECGs. The measurements were made manually using 0.01 second intervals. From each lead, QRS duration, $\mathrm{QT}_{\text {end }}$, and $\mathrm{QT}_{\text {apex }}$ intervals were measured for at least three complexes. $\mathrm{QT}_{\text {end }}$ interval was measured from the onset of the $\mathrm{Q}$ wave to the end of the $\mathrm{T}$ wave, defined as the return to the TP baseline. If the $\mathrm{T}$ wave was interrupted by a $U$ wave, the end of the $T$ wave was defined by principles outlined by Lepeschkin $e t a l .{ }^{16}$ If the end of the $\mathrm{T}$ wave could not be determined clearly, the lead was excluded from the analysis. $\mathrm{QT}_{\text {apex }}$ interval was measured from the onset of the $\mathrm{Q}$ wave to the apex of the $T$ wave, defined as the centre of the highest amplitude of the $T$ wave. The $T$ wave was considered biphasic if after the first $T$ wave apex there was a second T wave apex (of opposite polarity and an amplitude $\geqslant 0.1 \mathrm{mV}$ ) and the time interval between these was $\leqslant 150 \mathrm{~ms} .{ }^{16}$ Leads with biphasic $\mathrm{T}$ waves were excluded from analysis of $\mathrm{QT}_{\text {apex }}$ intervals (mean (SD) 1.1 (1.7) excluded leads for each ECG in the ventricular fibrillation group and 0.7 (1.3) leads in the control group). If less than six $\mathrm{QT}_{\text {apex }}$ intervals were measurable in the ECG, the $\mathrm{QT}_{\text {apex }}$ and related intervals $\left(\mathrm{JT}_{\text {apex }}\right.$ and $T_{\text {end }}$ ) were excluded from this ECG and its matched counterpart (one patient in both groups: two case control pairs). $\mathrm{JT}_{\text {end }}$ $\left(\mathrm{QT}_{\text {end }}-\mathrm{QRS}\right), \mathrm{JT}_{\text {apex }}\left(\mathrm{QT}_{\text {apex }}-\mathrm{QRS}\right)$, and $\mathrm{T}_{\text {end }}$ $\left(\mathrm{QT}_{\text {end }}-\mathrm{QT}_{\text {apex }}\right)$ intervals were calculated in each lead.

From the measurable leads in each ECG, the maximal QRS duration $\left(\mathrm{QRS}_{\max }\right)$ and maximal and minimal $\mathrm{QT}_{\text {end }}, \mathrm{QT}_{\text {apex }}, \mathrm{JT}_{\text {end }}, \mathrm{JT}_{\text {apex }}$, and $\mathrm{T}_{\text {end }}$ intervals were determined (referred to later as $\mathrm{QT}_{\text {end }}$ max, etc). Heart rate was determined from the RR intervals preceding the measured complexes. Heart rate correlated significantly with the QT and JT intervals ( $r$ values ranging from -0.65 to -0.73 ; $p$ values $<0.001)$. For heart rate correction we used the nomogram method. ${ }^{17}$ In the results, both measured and rate corrected $\mathrm{QT}$ and $\mathrm{JT}$ intervals are presented. The maximal and minimal $\mathrm{T}_{\text {end }}$ intervals correlated only weakly with heart rate $(r=-0.28$ and -0.25 , respectively) and were not rate corrected.

ECG measures of repolarisation dispersion The $\mathrm{QT}_{\text {end }}$ dispersion $\left(\mathrm{QT}_{\text {end }} \mathrm{max}-\mathrm{QT}_{\text {end }} \mathrm{min}\right)$ and respectively $\mathrm{QT}_{\text {apex }}$ dispersion, $\mathrm{JT}_{\text {end }}$ 
Table 1 Clinical characteristics of the study groups

\begin{tabular}{|c|c|c|c|}
\hline & $\begin{array}{l}\text { Controls } \\
(n=50)\end{array}$ & $\begin{array}{l}\text { VF group } \\
(n=50)\end{array}$ & $p$ value \\
\hline \multicolumn{4}{|l|}{ Matched characteristics } \\
\hline Sex (male/female) & $48 / 2$ & $48 / 2$ & \\
\hline Age (years) & $58(8)$ & $57(8)$ & \\
\hline $\mathrm{LV}$ ejection fraction (\%) & $41(16)$ & $41(18)$ & \\
\hline Number of diseased vessels & $2.4(0.8)$ & $2.4(0.7)$ & \\
\hline $1 / 2 / 3$ vessel CAD & $9 / 14 / 27$ & $8 / 16 / 26$ & \\
\hline Previous myocardial infarction & 39 & 39 & \\
\hline Inferior & 15 & 15 & \\
\hline Anterior & 13 & 13 & \\
\hline Multiple & 10 & 10 & \\
\hline Unknown & 1 & 1 & \\
\hline$\beta$ Blockers during study ECG & 23 & 21 & \\
\hline \multicolumn{4}{|l|}{ Other characteristics } \\
\hline History of hypertension & 19 & 21 & NS \\
\hline History of diabetes & 4 & 6 & NS \\
\hline NYHA class (I/II/III/IV) & $0 / 9 / 38 / 3$ & $4 / 33 / 12 / 1$ & $<0.001$ \\
\hline Positive exercise test & 45 & 24 & $<0.001$ \\
\hline Thrombolytic therapy & 17 & 6 & $<0.05$ \\
\hline Previous CABG & 3 & 8 & NS \\
\hline \multicolumn{4}{|l|}{ Number of previous MIs } \\
\hline $0 / 1 / 2 / 3$ & $11 / 25 / 10 / 3$ & $11 / 29 / 5 / 4$ & NS \\
\hline Left ventricular aneurysm & 2 & 12 & $<0.01$ \\
\hline Left ventricular hypertrophy $\star$ & 7 & 5 & NS \\
\hline \multicolumn{4}{|l|}{ Medication during study ECG } \\
\hline Digitalis & 12 & 13 & NS \\
\hline Diuretics & 15 & 20 & NS \\
\hline Calcium channel blockers $\dagger$ & 23 & 5 & $<0.001$ \\
\hline
\end{tabular}

Data are number of individuals or mean (SD).

${ }^{\star} \mathrm{SV}_{1}+\mathrm{RV}_{5} \geq 35 \mathrm{~mm}$ in the ECG; †Verapamil, diltiatzem or nifedipine.

dispersion, $\mathrm{JT}_{\text {apex }}$ dispersion, and $\mathrm{T}_{\text {end }}$ dispersion were calculated for each ECG (referred to later as $\mathrm{QT}_{\text {end }}$ dispersion, etc). None of these dispersion measures correlated with heart rate in the ventricular fibrillation or control groups (with $r$ values ranging from 0.04 to -0.14$)$. Therefore, the dispersion measures were not corrected for heart rate. For each ECG, standard deviations for $\mathrm{QT}_{\text {end }}, \mathrm{QT}_{\text {apex }}$, $\mathrm{JT}_{\text {end }}, \mathrm{JT}_{\text {apex }}$ and $\mathrm{T}_{\text {end }}$ intervals in all the measurable leads were calculated (referred to later as $\mathrm{QT}_{\text {end }} \mathrm{SD}$, etc).

\section{STATISTICAL ANALYSIS}

The paired samples $t$ test was used to compare the two study groups for continuous variables. Either Pearson's or Spearman's correlation coefficients were used in determining univariate correlations between continuous variables. Chi-squared test with Yates's contingency correction or Fisher's exact test were used for categorical variables when appropriate. Significance was set at a two tailed $\mathrm{p}$ value $<0.05$.
Table 2 RR interval, $Q R S$ duration, and repolarisation intervals

\begin{tabular}{llll}
\hline & $\begin{array}{l}\text { Controls } \\
(n=50)\end{array}$ & $\begin{array}{l}\text { VF group } \\
(n=50)\end{array}$ & p value \\
\hline RR interval & $977(166)$ & $974(186)$ & $\mathrm{NS}$ \\
QRS duration & $111(17)$ & $126(27)$ & $<0.001$ \\
Repolarisation intervals & & & \\
$\mathrm{QT}_{\text {apex }}$ & $338(33)$ & $362(42)$ & $<0.01$ \\
$\mathrm{QT}_{\text {end }}$ & $420(41)$ & $439(46)$ & $<0.05$ \\
$\mathrm{JT}_{\text {apex }}$ & $241(33)$ & $253(37)$ & $\mathrm{NS}$ \\
$\mathrm{JT}_{\text {end }}$ & $320(40)$ & $327(41)$ & $\mathrm{NS}$ \\
$\mathrm{T}_{\text {end }}$ & $101(16)$ & $104(17)$ & $\mathrm{NS}$ \\
$\mathrm{Heart}_{\text {rate corrected repolarisation intervals }}$ & \\
$\mathrm{QT}_{\text {apex }}$ & $344(24)$ & $368(30)$ & $<0.001$ \\
$\mathrm{QT}_{\text {end }}$ & $426(30)$ & $446(34)$ & $<0.01$ \\
$\mathrm{JT}_{\text {apex }}$ & $247(21)$ & $259(26)$ & $<0.05$ \\
$\mathrm{JT}_{\text {end }}$ & $326(28)$ & $334(29)$ & $\mathrm{NS}$ \\
\hline
\end{tabular}

Values are mean (SD) in ms.

\section{Results}

CLINICAL CHARACTERISTICS

Table 1 shows the clinical characteristics of the study groups. Nineteen patients in the ventricular fibrillation group were inducible to sustained monomorphic ventricular tachycardia.

ELECTROCARDIOGRAPHIC DATA

The mean (SD) number of measured $\mathrm{QT}_{\text {end }}$ intervals for each ECG was $11.2(0.9)$ in the ventricular fibrillation group and $11.0(0.9)$ in the control group (not significant). The mean (SD) number of measured $\mathrm{QT}_{\text {apex }}$ intervals for each ECG was 10.1 (1.5) in the ventricular fibrillation group and 10.4 (1.3) in the control group (not significant).

The mean (SD) heart rate was 64 (14) in the ventricular fibrillation group and 63 (11) in the control group. QRS ${ }_{\max }$ was significantly longer in the ventricular fibrillation group than in the control group (table 2). Both maximal $\mathrm{QT}_{\text {apex }}$ and $\mathrm{QT}_{\text {end }}$ intervals were longer in the ventricular fibrillation group than in the control group (table 2).

ELECTROCARDIOGRAPHIC MEASURES OF REPOLARISATION DISPERSION

$\mathrm{QT}_{\text {apex }}$ dispersion, $\mathrm{QT}_{\text {apex }} \mathrm{SD}, \mathrm{T}_{\text {end }}$ dispersion, and $\mathrm{T}_{\text {end }} \mathrm{SD}$ were greater in the ventricular fibrillation group compared with the control group (table 3). The measured values for $\mathrm{QT}_{\text {apex }}$ and $T_{\text {end }}$ dispersion are shown in fig 1 , which illustrates a considerable overlap between the

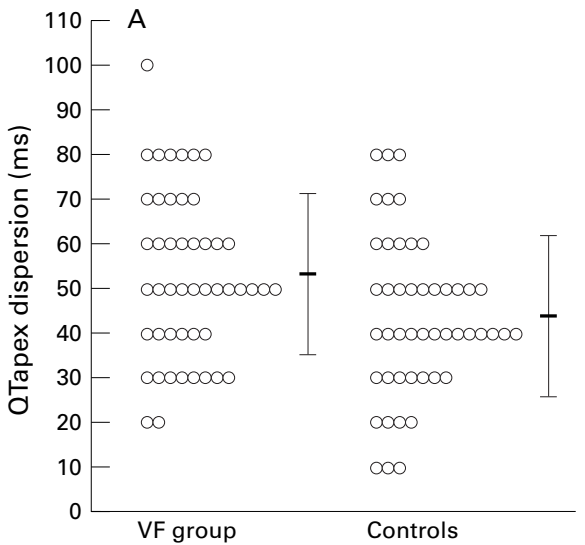

Figure 1 (A) $Q T$ dispersion and (B) $T$ dispersion in patients prone to ventricular fibrillation (VF) compared with controls. Dispersion values of each patient in the study groups are depicted. Thick horizontal lines are the group mean values and vertical lines are $1 S D$. 
Table 3 Electrocardiographic measures of repolarisation dispersion

\begin{tabular}{llll}
\hline $\begin{array}{l}\text { Repolarisation } \\
\text { dispersion }\end{array}$ & $\begin{array}{l}\text { Controls } \\
(n=50)\end{array}$ & $\begin{array}{l}\text { VF group } \\
(n=50)\end{array}$ & p value \\
\hline $\mathrm{QT}_{\text {apex }} \mathrm{D}$ & $44(18)$ & $53(18)$ & $<0.01$ \\
$\mathrm{QT}_{\text {end }} \mathrm{D}$ & $49(20)$ & $55(25)$ & $\mathrm{NS}$ \\
$\mathrm{JT}_{\text {apex }} \mathrm{D}$ & $50(16)$ & $56(19)$ & 0.05 \\
$\mathrm{JT}_{\text {end }} \mathrm{D}$ & $50(18)$ & $57(22)$ & $\mathrm{NS}$ \\
$\mathrm{T}_{\text {end }} \mathrm{D}$ & $38(15)$ & $46(17)$ & $<0.05$ \\
$\mathrm{QT}_{\text {apex }} \mathrm{SD}$ & $14(6)$ & $17(7)$ & $<0.01$ \\
$\mathrm{QT}_{\text {end }} \mathrm{SD}$ & $16(7)$ & $18(9)$ & $\mathrm{NS}$ \\
$\mathrm{JT}_{\text {apex }} \mathrm{SD}$ & $16(5)$ & $18(7)$ & $\mathrm{NS}$ \\
$\mathrm{JT}_{\text {end }} \mathrm{SD}$ & $16(6)$ & $18(8)$ & $\mathrm{NS}$ \\
$\mathrm{T}_{\text {end }} \mathrm{SD}$ & $13(5)$ & $16(6)$ & $<0.05$ \\
\hline
\end{tabular}

Values are mean (SD) in ms.

$\mathrm{D}$, maximum - minimum of the respective intervals; SD, standard deviation of the respective intervals.

two groups. $\mathrm{QRS}_{\max }$ correlated with $\mathrm{QT}_{\text {apex }}$ dispersion both in the ventricular fibrillation group $(r=0.36 ; \mathrm{p}<0.05)$ and in the control group $(r=0.51 ; \mathrm{p}<0.001)$, but did not correlate with $\mathrm{T}_{\text {end }}$ dispersion in either study group. $\mathrm{QT}_{\text {apex }}$ dispersion and $\mathrm{T}_{\text {end }}$ dispersion did not correlate significantly in either study group $(r=0.26$ and 0.18 in the ventricular fibrillation and control groups, respectively).

CORRELATIONS OF $\mathrm{QT}_{\mathrm{APEX}}$ AND $\mathrm{T}_{\mathrm{END}}$ DISPERSIONS IN COMBINED PATIENT GROUPS

The patient groups differed with regard to the use of calcium channel blockers, positive exercise tests, previous thrombolytic therapy, and left ventricular aneurysms. To assess the effect of these variables on $\mathrm{QT}_{\text {apex }}$ and $\mathrm{T}_{\text {end }}$ dispersions, combined patient groups were divided into two groups on the basis of these variables. There was no difference in mean (SD) $\mathrm{QT}_{\text {apex }}$ dispersion in patients with or without calcium channel blockers (46 (19) ms $v 49$ (19) ms, respectively; $\mathrm{p}=0.5$ ) or in mean (SD) $\mathrm{T}_{\text {end }}$ dispersion in patients with or without these drugs (45 (19) ms v 41 (16) ms, respectively; $\mathrm{p}=0.4)$. There was no significant difference in mean (SD) $\mathrm{QT}_{\text {apex }}$ dispersion in patients with positive exercise tests compared with those with negative exercise tests (46 (19) ms v 53 (19) ms, respectively; $\mathrm{p}=0.1$ ) or in mean (SD) $\mathrm{T}_{\text {end }}$ dispersion (41 (17) $v 43$ (16) ms, respectively; $p=0.4$ ). Patients who had received thrombolytic treatment had less $T_{\text {end }}$ dispersion compared with those who had not (33 (14) v 45 (16) ms, respectively; $\mathrm{p}<0.01$ ), but there was no difference in mean (SD) $\mathrm{QT}_{\text {apex }}$ dispersion (49 (17) v 48 (19) ms, respectively; $p=0.7)$. Patients with a left ventricular aneurysm showed greater $\mathrm{QT}_{\text {apex }}$ dispersion compared with those without an aneurysm (59 (17) v 46 (18) ms, respectively; $\mathrm{p}<0.05)$, but there was no difference in mean (SD) $\mathrm{T}_{\text {end }}$ dispersion (43 (13) v 42 (17) $\mathrm{ms}$, respectively; $\mathrm{p}=0.5)$.

CORRELATIONS OF DISPERSION MEASURES IN THE VENTRICULAR FIBRILLATION GROUP

To assess the potential influence of the mechanism of ventricular fibrillation on dispersion measures, the ventricular fibrillation group was divided into two subgroups on the basis of a positive or negative exercise test for ischaemia. There was no significant difference in any of the dispersion measures between
Table 4 Electrocardiographic measures of repolarisation dispersion in patients with ventricular fibrillation with a previous myocardial infarction with or without inducible sustained monomorphic ventricular tachycardia

\begin{tabular}{lll}
\hline & $\begin{array}{l}S M V T(-) \\
(n=20)\end{array}$ & $\begin{array}{l}S M V T(+) \\
(n=19)\end{array}$ \\
\hline $\begin{array}{ll}\text { QRS duration } \\
\text { Repolarisation dispersion }\end{array}$ & $132(32)$ & $131(24)$ \\
$\mathrm{QT}_{\text {apex }} \mathrm{D}$ & $58(20)$ & $53(17)$ \\
$\mathrm{QT}_{\text {end }} \mathrm{D}$ & $61(30)$ & $58(20)$ \\
$\mathrm{JT}_{\text {apex }} \mathrm{D}$ & $57(20)$ & $58(20)$ \\
$\mathrm{JT}_{\text {end }} \mathrm{D}$ & $57(25)$ & $62(21)$ \\
$\mathrm{T}_{\text {end }} \mathrm{D}$ & $46(16)$ & $46(18)$ \\
$\mathrm{QT}_{\text {apex }} \mathrm{SD}$ & $19(7)$ & $18(7)$ \\
$\mathrm{QT}_{\text {end }} \mathrm{SD}$ & $20(12)$ & $19(5)$ \\
$\mathrm{JT}_{\text {apex }} \mathrm{SD}$ & $19(8)$ & $19(7)$ \\
$\mathrm{JT}_{\text {end }} \mathrm{SD}$ & $19(10)$ & $20(7)$ \\
$\mathrm{T}_{\text {end }} \mathrm{SD}$ & $16(6)$ & $15(6)$
\end{tabular}

Values are mean (SD) in ms; $p$ values not significant for any parameter.

$\mathrm{D}$, maximum - minimum of the respective intervals; SD, standard deviation of the respective intervals; SMVT, sustained monomorphic ventricular tachycardia $(+)$ inducible or $(-)$ not inducible in the electrophysiological study.

these subgroups. Mean (SD) $\mathrm{QT}_{\text {apex }}$ dispersion was 54 (17) $\mathrm{ms}$ in patients without exercise ischaemia and $52(21) \mathrm{ms}$ in patients with exercise ischaemia $(\mathrm{p}=0.7)$; mean $(\mathrm{SD}) \mathrm{T}_{\text {end }}$ dispersion was 45 (16) $\mathrm{ms}$ and 47 (18) $\mathrm{ms}$, respectively $(\mathrm{p}=0.6)$. Furthermore, ventricular fibrillation patients with a previous myocardial infarction were divided into two subgroups on the basis of inducibility of sustained monomorphic ventricular tachycardia in the electrophysiological study. There was no significant difference in any of the dispersion measures between the subgroups (table 4 ).

\section{Discussion}

Our results show that $\mathrm{QT}_{\text {apex }}$ dispersion and $\mathrm{T}_{\text {end }}$ dispersion are increased in coronary artery disease patients prone to ventricular fibrillation, even when the extent of the coronary heart disease and use of $\beta$ blockers are taken into consideration. These observations support the role of non-homogenous repolarisation as a contributing factor to ventricular fibrillation. However, because there was a considerable overlap between the groups studied, repolarisation dispersion assessed by these measures from a 12 lead ECG is not a clinically useful method for identifying individual coronary artery disease patients at risk of sudden cardiac death.

QT DISPERSION AND VENTRICULAR FIBRILLATION In a recent prospective study of a coronary risk population, we found that $\mathrm{QT}_{\text {apex }}$ dispersion separated patients prone to sudden cardiac death from controls, although $\mathrm{QT}_{\text {end }}$ dispersion did not. ${ }^{18}$ In this study, increased $\mathrm{QT}_{\text {apex }}$ dispersion was associated with susceptibility to ventricular fibrillation, even when the extent of heart disease and the use of $\beta$ blockers were taken into consideration. During sinus rhythm, the probable cause of $T$ waves are transmural gradients of repolarisation. ${ }^{19}$ An increase in transmural non-homogeneity of repolarisation might influence the spatial differences in the timing of the apex of the $T$ wave, thus increasing $\mathrm{QT}_{\text {apex }}$ dispersion. Experiments by El-Sherif et al have shown that the dispersion of the intramyocardial rather than endocardial or 
epicardial action potential duration is critical for polymorphic ventricular tachycardia. ${ }^{20} \mathrm{On}$ the other hand, left ventricular hypertrophy creates dispersion of repolarisation, ${ }^{21}$ and is associated with increased $\mathrm{QT}_{\text {apex }}$ dispersion. ${ }^{22}$ After a myocardial infarction the non-infarcted myocardium undergoes significant hypertrophy. ${ }^{23}$ Dispersion of refractory periods in myocardial regions remote from the infarction might be relevant to postinfarction ventricular fibrillation. ${ }^{24}$ It is possible that hypertrophy of the non-infarcted myocardium might increase $\mathrm{QT}_{\text {apex }}$ dispersion, both of which are associated with the risk of ventricular fibrillation after myocardial infarction. In this retrospective study, hypertrophy of the noninfarcted left ventricular myocardium was not assessed. The conventional electrocardiographic index of left ventricular hypertrophy (Sokolow-Lyon: $\mathrm{SV}_{1}+\mathrm{RV}_{5}>35 \mathrm{~mm}$ ) did not differ between the study groups (table 1 ).

$\mathrm{T}_{\text {end }}$ DISPERSION AND VENTRICULAR FIBRILLATION The dispersion of repolarisation is determined by the combined effects of differences in activation times and action potential durations. ${ }^{25}$ Patients prone to ventricular fibrillation had longer QRS complexes than controls. The entire QRS duration has to be accounted for by differences in activation times. ${ }^{26}$ In our study, $T_{\text {end }}$ dispersion was increased in patients prone to ventricular fibrillation, whereas $\mathrm{QT}_{\text {end }}$ and $\mathrm{JT}_{\text {end }}$ interval dispersions did not differ significantly. In an experimental model, Zabel et al observed that $\mathrm{T}_{\text {end }}$ interval, total $\mathrm{T}$ wave area, and late $\mathrm{T}$ wave area reflected the dispersion of action potential durations better than $\mathrm{QT}_{\text {end }}$ and $\mathrm{JT}_{\text {end }}$ dispersions. ${ }^{7}$ In our study, QRS duration and $\mathrm{T}_{\text {end }}$ dispersion did not correlate significantly. Therefore, prolonged QRS duration and increased $T_{\text {end }}$ dispersion, both contributors of repolarisation dispersion, might serve as independent markers of susceptibility to ventricular fibrillation in coronary artery disease.

CORRELATIONS OF THE DISPERSION MEASURES The objective of this study was to examine whether increased electrocardiographic measures of repolarisation dispersion are associated with susceptibility to ventricular fibrillation, which is the most common cause of sudden cardiac death. The ventricular fibrillation group in this study corresponds to previous descriptions of sudden cardiac death populations in terms of percentage of patients inducible to sustained monomorphic ventricular tachycardia in the electrophysiological study and left ventricular ejection fraction. ${ }^{1}$ The mechanism of ventricular fibrillation in coronary artery disease is heterogenous. These patients might have developed ventricular fibrillation as a primary arrhythmia from electrical instability, secondary to ventricular tachycardia or on an ischaemic basis. However, subgroup analysis of ventricular fibrillation patients with or without a positive exercise test for ischaemia, or ventricular fibrillation patients with a previous myocardial infarction with or without inducible sustained monomor- phic ventricular tachycardia, revealed no significant differences in dispersion parameters between the subgroups.

Patients with a ventricular aneurysm exhibited greater $\mathrm{QT}_{\text {apex }}$ dispersion compared to those without an aneurysm. The observed difference might be a reflection of the left ventricular regional shortening abnormalities. Whether such a difference in $\mathrm{QT}_{\text {apex }}$ dispersion contributes to susceptibility to ventricular fibrillation or is merely an epiphenomenon of an aneurysm is unknown.

\section{STUDY LIMITATIONS}

The control patients were matched with the patients in the ventricular fibrillation group with regard to the number of the diseased coronary vessels, left ventricular ejection fraction, and the use of $\beta$ blockers. Nonetheless, patients in the ventricular fibrillation group were in lower NYHA functional classes and had positive exercise tests less often. Because the controls were matched individually for several variables, it would have been unlikely for us to find suitable control patients in low NYHA classes without signs of exercise ischaemia who would have undergone angiography. As ischaemia itself increases dispersion of repolarisation, ${ }^{27}$ our results between the study groups in repolarisation dispersion measures should not be overestimates in this respect. Matching for $\beta$ blockers also resulted in an imbalance in the use of calcium channel blockers. When the combined groups were divided on the basis of the use of calcium channel blockers, there were no significant differences in any of the ECG interval or dispersion measurements.

In the case of biphasic $T$ waves, it is not clear which $\mathrm{T}$ wave deflection should be defined as the $T_{\text {apex }}$. Therefore, we decided ad hoc to exclude the biphasic $\mathrm{T}$ waves from $\mathrm{QT}_{\text {apex }}$ and related ( $\mathrm{JT}_{\text {apex }}$ and $\mathrm{T}_{\text {end }}$ ) measurements. A bias is unlikely because, on average, there was only one biphasic $T$ wave for each ECG, and there was no significant difference in the number of biphasic $\mathrm{T}$ waves between the study groups.

Successful thrombolytic treatment reduces $\mathrm{QT}_{\text {end }}$ dispersion. ${ }^{28}$ More of our controls had received thrombolytic treatment than patients in the ventricular fibrillation group. However, judged by appearance of Q waves in the ECG and lowered left ventricular ejection fraction, few treatments had been successful. When the groups were combined, there was no difference in $\mathrm{QT}_{\text {end }}$ dispersion in patients who had previously received thrombolytic treatment compared with those who had not. Nonetheless, previous thrombolytic treatment was associated with reduced $\mathrm{T}_{\text {end }}$ dispersion.

\section{CONCLUSIONS}

Conventional $\mathrm{QT}_{\text {end }}$ dispersion does not separate coronary artery disease patients with a history of ventricular fibrillation from matched controls. Instead, $\mathrm{QT}_{\text {apex }}$ and $\mathrm{T}_{\text {end }}$ dispersions separate patients prone to ventricular fibrillation even from controls carefully matched for the extent of the underlying coronary artery disease and the use of $\beta$ blockers. Because of 
considerable overlap between the groups, QT dispersion does not provide clinically useful information on the risk of ventricular fibrillation if the features of the coronary heart disease obtained from invasive studies are known. The differences detected in this study support the association between non-homogenous repolarisation and susceptibility to ventricular fibrillation. To obtain clinically useful information, more accurate methods are needed for assessing ventricular repolarisation dispersion.

This study was supported financially by the Finnish Foundation for Cardiovascular Research.

1 Osswald S, Trouton TG, O'Nunain S, et al. Patients with cardiac arrest and documented ventricular fibrillation. In Akhtar M, Myerburg RJ, Ruskin NJ, eds. Sudden cardiac death. Prevalence, mechanisms, and approaches to diagnosis and management. Malvern: Williams and Wilkins, 1994:46585.

2 Han J, Moe GK. Nonuniform recovery of excitability in ventricular muscle. Circ Res 1964;14:44-60.

3 Merx W, Yoon MS, Han J. The role of local disparity in conduction and recovery time on ventricular vulnerability to fibrillation. Am Heart f 1977;94:603-10.

4 Kuo C-S, Munakata K, Reddy P, et al. Characteristics and possible mechanism of ventricular arrhythmia dependent of the dispersion of action potential duration. Circulation 1983;67:1356-67.

5 Lee RJ, Liem LB, Cohen TJ, et al. Relation between repolarization and refractoriness in the human ventricle: cycle length dependence and

6 Day CP, McComb JM, Campbell RWF. QT dispersion: an indication of arrhythmia risk in patients with long QT indication of arrhythmia risk in pati

7 Zabel M, Portnoy S, Franz MR. Electrocardiographic indexes of dispersion of ventricular repolarization: an isolated heart validation study. F Am Coll Cardiol 1995; 25:746-52

8 Higham PD, Hilton CJ, Aitcheson JD, et al. QT dispersion: a measure of underlying dispersion of ventricular recovery times [abstract]. Circulation 1992;86(suppl I):I-392.

9 Buja G, Miorelli M, Turrini P, et al. Comparison of QT dispersion in hypertrophic cardiomyopathy between patients with and without ventricular arrhythmias and sudden death. Am f Cardiol 1993; 72:973-6.

10 Tieleman RG, Crijns HJGM, Wiesfeld ACP, et al. Increased dispersion of refractoriness in the absence of QT prolongation in patients with mitral valve prolapse and ventricular tion in patients with mitral valve prolapse

11 Daliento L, Caneve F, Turrini P, et al. Clinical significance of high-frequency, low-amplitude electrocardiographic sighigh-frequency, low-amplitude electrocardiographic signals and QT dispersion in patients operated

12 Gatzoulis MA, Till JA, Redington AN. Depolarizationrepolarization inhomogeneity after repair of tetralogy of
Fallot. The substrate for malignant ventricular tachycardia? Circulation 1997;95:401-4.

13 Van de Loo A, Arendts W, Hohnloser SH. Variability of QT dispersion measurements in the surface electrocardiogram in patients with acute myocardial infarction and in normal subjects. Am f Cardiol 1994;74:1113-18.

14 Perkiömäki JS, Koistinen J, Yli-Mäyry S, et al. Dispersion of QT interval in patients with and without susceptibility to ventricular tachyarrhythmias after previous myocardial infarction. 7 Am Coll Cardiol 1995;26:174-9.

15 Zareba W, Moss AJ, le Cessie S. Dispersion of ventricular repolarization and arrhythmic cardiac death in coronary artery disease. Am f Cardiol 1994;74:550-3.

16 Lepeschkin E, Surawicz B. The measurement of the QT interval of the electrocardiogram. Circulation 1952;6:37888.

17 Karjalainen J, Viitasalo $M$, Mänttäri $M$, et al. Relation between QT intervals and heart rates from 40 to 120 beats/ min in rest electrocardiograms of men and a simple method min in rest electrocardiograms of men and a simple method to adjust

18 Mänttäri M, Oikarinen L, Manninen V, et al. QT dispersion as a risk factor for sudden cardiac death and fatal myocardial infarction in a coronary risk population. Heart 1997;78:268-72.

19 Franz MR, Bargheer K, Rafflenbeul W, et al. Monophasic action potential mapping in human subjects with normal electrocardiograms: direct evidence for the genesis of the $\mathrm{T}$ wave. Circulation 1987;75:379-86.

20 El-Sherif N, Zeiler RH, Craelius W, et al. QTU prolongation and polymorphic ventricular tachyarrhythmias due to bradycardia-dependent early after depolarizations. Circ Res 1988;63:286-315.

21 Kowey PR, Friehling TD, Sewter J, et al. Electrophysiological effects of left ventricular hypertrophy: effect of calcium and potassium channel blockade. Circulation 1991;83: 2067-75.

22 Perkiömäki JS, Ikäheimo MJ, Pikkujämsä SM, et al. Dispersion of the QT interval and autonomic modulation of heart rate in hypertensive men with and without left ventricular hypertrophy. Hypertension 1996;28:16-21.

23 Anversa P, Beghi C, Kikkawa Y, et al. Myocardial infarction in rats: infarct size, myocyte hypertrophy, and capillary growth. Circ Res 1986;58:26-37.

24 Misier ARR, Opthof T, van Hemel NM, et al. Dispersion of "refractoriness" in noninfarcted myocardium of patients myocardial infarction. Circulation 1995;91:2566-72.

25 Surawicz B. Dispersion of refractoriness in experimental and clinical arrhythmias. In: Surawicz B, ed. Electrophysiologic basis of ECG and cardiac arrhythmias. Malvern: Williams and Wilkins, 1995:242-67.

26 Franz MR, Bargheer K, Costard-Jäckle A, et al. Human ventricular repolarization and T wave genesis. Prog Cardiovasc Dis 1991;33:369-84.

27 Sporton SC, Taggart P, Sutton PM, et al. Acute ischaemia: a dynamic influence on QT dispersion. Lancet 1997;349: 306-9.

28 Moreno FL, Villanueva T, Karagounis LA, et al. Reduction in QT interval dispersion by successful thrombolytic therapy in acute myocardial infarction. Circulation 1994;90: 94-100. 